\title{
Determinación de Razas Fisiológicas y Tipo de Apareamiento en Aislamientos de Phytophthora infestans (Mont) De Bary
}

\author{
Juan Bautista López ${ }^{1}$ María Elena Márquez, ${ }^{1}$ Sonia Jaramillo, ${ }^{2}$ José \\ Luís Zapata, ${ }^{3}$ John Jairo Mazo, ${ }^{2}$ y Luis Fernando Patiño ${ }^{2}$
}

\section{Resumen}

El problema más limitante del cultivo de la papa en muchas regiones del mundo, lo constituye la gota o tizón tardío ocasionado por el hongo Phytophthora infestans. Esta investigación se realizó con el objetivo de determinar variaciones poblacionales de este patógeno, basadas en el tipo de apareamiento y en la presencia de razas fisiológicas en 19 aislamientos de $P$. infestans obtenidos en plantas de papa (Solanum tuberosum) variedades Diacol Capiro, ICA Puracé e ICA Cumanday; papa criolla (Solanum phureja), pepino (Solanum muricatum) y tomate (Lycopersicon esculentum) en diferentes zonas del Departamento de Antioquia (Colombia).

Los resultados obtenidos a partir de un apareamiento patrón $A_{1}$, con los aislamientos colectados en las diferentes zonas sugieren la existencia de una población de $P$. infestans con un solo tipo de apareamiento. La prueba de virulencia del hongo, realizada en folíolos de 11 diferenciales de papa mostró la presencia de 6 razas fisiológicas, de las cuales la más frecuente fue la $7446(58 \%)$, con los factores de virulencia 1-2-3-4-7-10-11. Aislamientos monozoospóricos presentaron los mismos factores de virulencia que sus respectivos aislamientos recolectados en el campo, lo que sugiere homogeneidad de la población fungosa. La gran

1 Laboratorio de Genética y Biología Molecular, Departamento de Biología. Facultad de Ciencias Agropecuarias, Universidad Nacional de Colombia, Sede Medellín

2 Laboratorio de Fisiología de Crecimiento y Desarrollo de las Plantas. Facultad de Ciencias Agropecuarias, Universidad Nacional de Colombia, Sede Medellin.

3 Laboratorio Fitopatología CIP-CORPOICA, La Selva, Rionegro Antioquia, Colombia. 
Aceptado para publicación: mayo 1997.

complejidad de las razas fisiológicas de $P$. infestans podría estar influenciada por diversos factores genéticos y medioambientales.

\author{
Palabras claves adicionales: tizón tardío de la papa, Phytophthora \\ infestans, tipo de apareamiento, razas \\ fisiológicas.
}

\title{
Determination of Physiological Races and Mating Types in isolates of Phytophthora infestans (Mont) De Bary
}

\begin{abstract}
Summary
The fungus Phytophthora infestans (Mont) de Bary causes late blight which is the most serious obstacle to potato cultivation in the world. In this study, the mating type and physiological races were determined in $19 \mathrm{P}$. infestans isolates from Solanum phureja, Solanum muricatum, Lycopersicon esculentum and three Solanum tuberosum cultivars (Diacol Capiro, ICA Puracé and ICA Cumanday). The results showed the presence of mating type $A_{1}$, six physiological races and a virulence factor average of 6.6. This study shows a great homogeneity and complexity in $P$. infestans populations collected in the Department of Antioquia (Colombia).
\end{abstract}

Additional Index words: late blight, Phytophthora infestans, mating type, physiological races.

\section{Introducción}

La papa se ha constituido en una de las principales fuentes de alimento para el hombre. Sin embargo el cultivo de esta planta es severamente limitado por la enfermedad conocida como tizón tardío o gota, causada por Phytophthora infestans (Mont.) de Bary, enfermedad que puede devastar en corto tiempo los campos cultivados, llegando incluso a provocar grandes catástrofes de carácter socioeconómico. 
Los reportes existentes sugieren que el origen del patosistema Phytophthora infestans/Solanum tuberosum es el Valle de Toluca en México (Tooley et al, 1985; Fry et al, 1989), lo cual es corroborado por los hallazgos de numerosos genes de resistencia al hongo, en especies silvestres de Solanum originarias de esa región (Niederhauser y Mills, 1953). Otro argumento para fortalecer estas suposiciones es la presencia en igual frecuencia de los dos tipos de apareamiento $A_{1}$ y $A_{2}$ del estado sexual del patógeno (Gallegly and Galindo, 1958; Galindo y Gallegly, 1960).

En la actualidad diversos factores pueden incrementar la patogenicidad del hongo. Entre ellos están la migración a nivel mundial de los dos tipos de apareamiento de $P$. infestans, lo cual implica una reproducción sexual que posiblemente incrementa la variabilidad genética, que da como consecuencia un amplio espectro de posibilidades ante nuevas condiciones que hacen más difícil el control químico y cultural de la enfermedad (Henfling, 1987).

Otro factor clave en la variación poblacional de $P$. Infestans lo constituye el cultivo continuo de variedades de papa con los llamados genes mayores. Estos pueden contribuir a seleccionar razas patogénicas del hongo que pueden manifestarse con una aparente mayor agresividad frente a cultivos susceptibles (Henfling, 1987).

Desde que se determinó la presencia de oosporas de $P$. infestans en México se ha aceptado de forma general que el tipo $A_{1}$ es el que está ampliamente distribuido, y que el tipo $A_{2}$ sólo estaba confinado a México Central. A mediados de la década del 80, el tipo $A_{2}$ fue encontrado en localidades diferentes al Valle de Toluca (Hohl e Iselin, 1984), en regiones cultivadas con papa donde se presenta la enfermedad (Daggett et al., 1993).

En lo que corresponde a las colecciones de $P$. infestans que contienen aislamientos $A_{2}$ muestreados antes de 1980 's, se tienen dos hipótesis: la primera, sugiere que estos aislamientos han sido introducidos de regiones donde está presente el tipo $A_{2}$ (Shaw, 1987). Y la segunda, propone que el tipo de apareamiento $A_{2}$ ha estado presente en estas regiones, pero no había sido detectado (Shattock et al, 1990).

La región de los Andes en Suramérica se ha sugerido como una de las localidades donde podría encontrarse el estado sexual de $P$. infestans, dado que esta zona es considerada el centro de origen de la papa cultivada, además de la existencia de una gran variabilidad de especies de plantas pertenecientes al género Solanum (Gallegly y Galindo, 1958). No obstante, las poblaciones de este patógeno en Suramérica parecen corresponder a un solo tipo de apareamiento $A_{1}$ (Zachman, 1976; Tooley et al., 1989) (Tabla 4). 
El número de factores de virulencia de $P$. infestans sería diferente en las diversas regiones del mundo, siendo México Central la que presenta mayor complejidad (Tooley et al., 1986), el Reino Unido de complejidad intermedia (Fry y Spielman, 1991), relativamente baja en Estados Unidos y Canadá (Tooley et al., 1986) y la más baja de todas en el Perú (Tooley et al., 1989).

El objetivo de la presente investigación fue evaluar las razas fisiológicas y la posible presencia del estado sexual de $P$. infestans en diferentes localidades del Departamento de Antioquia, con el fin de estimar la estructura poblacional del hongo y su implicación en el mejoramiento de la papa para resistencia al tizón tardío.

\section{Materiales y Métodos}

\section{Obtención de folíolos infectados y cultivos primarios}

Las recolecciones de folíolos infectados de tizón se efectuaron en 10 zonas diferentes del Departamento de Antioquia sobre Solanáceas como la papa (Solanum tuberosum), cultivares Diacol Capiro, ICA-Puracé e ICACumanday; papa criolla (Solanum phureja), el pepino (Solanum muricatum) y el tomate (Lycopersicon esculentum). Los datos de cada una de las muestras se registraron en la Tabla 1. Los aislamientos estudiados fueron recolectados entre diciembre de 1994 y junio de 1995. Los folíolos afectados de la planta de papa se lavaron con agua, se secaron a la sombra y se cortaron en trozos pequeños que contenían parte de la zona afectada. Sobre estos trozos se colocaron rodajas de papa de la variedad Tuquerreña en placas de Petri estériles. 
Tabla 1, Registro de muestras de P. infestans colectadas en el Departamento de Antioquia, Colombia

\begin{tabular}{|c|c|c|c|c|}
\hline Aislamiento & msnm & Municipio & $\begin{array}{c}\text { Edad } \\
\text { (meses) }\end{array}$ & Hospedantes \\
\hline$U_{1}$ & 2450 & La Unión & 1.5 & Diacol Capiro** \\
\hline $\mathrm{U}_{2}$ & 2460 & La Unión & 3.5 & Diacol Capiro** \\
\hline $\mathrm{U}_{3}$ & 2460 & La Unión & 3.0 & Solanum phureja \\
\hline $\mathrm{C}_{4}$ & 2140 & El Carmen & 1.5 & Diacol Capiro** \\
\hline $\mathrm{G}_{5}$ & 2300 & Guarne & 2.0 & Diacol Capiro** \\
\hline $\mathrm{UN}_{6}$ & 2550 & Santa Elena & 3.0 & Diacol Capiro** \\
\hline $\mathrm{UN}_{8}$ & 2550 & Santa Elena & 3.5 & ICA Puracé ${ }^{* *}$ \\
\hline $\mathrm{SV}_{7}$ & 2150 & San Vicente & 2.5 & Diacol Capiro" \\
\hline$E_{9}$ & 2100 & Bello & 3.0 & Diacol Capiro** \\
\hline$E_{10}$ & 2100 & Bello & 3.0 & Solanum phureja \\
\hline $\mathrm{ER}_{13}$ & 2300 & Entremos & 2.0 & Diacol Capiro** \\
\hline $\mathrm{SP}_{11}$ & 2500 & San Pedro & 2.5 & Solanum phureja \\
\hline $\mathrm{SP}_{12}$ & 2450 & San Pedro & 2.0 & Cumanday \\
\hline $\mathrm{SR}_{14}$ & 2540 & Santa Rosa & 2.0 & Diacol Capiro** \\
\hline $\mathrm{SR}_{15}$ & 2550 & Santa Rosa & 3.5 & Diacol Capiro** \\
\hline $\mathrm{SS}_{16}$ & 2400 & Sonsón & 3.0 & Diacol Capiro** \\
\hline $\mathrm{SS}_{17}$ & 2120 & Sonsón & 3.0 & Solanum phureja \\
\hline$P_{18}$ & 2120 & Rionegro & NO & Solanum murícatum \\
\hline $\mathrm{T}_{19}$ & 2120 & Rionegro & ND & Lycopersicon esculentum \\
\hline
\end{tabular}

${ }^{* *}$ Variedades de Solanum tuberosum. $\quad N D=$ no determinado

El cultivo se mantuvo a $90 \%$ de humedad relativa y a una temperatura entre $16-18^{\circ} \mathrm{C}$ durante 5 - 6 días en condiciones de cámara de crecimiento, situación registrada mediante higrotermógrafo, al término de lo cual se presenta la mayor esporulación del hongo. Luego se cosecharon los esporangios, mediante filtrado con tela (tul) para separar micelio y partículas diferentes, luego se filtró con tela miliporo para retener los esporangios y se indujo la liberación de zoosporas en agua estéril a $4^{\circ} \mathrm{C}$ por 30 a 60 minutos aproximadamente.

\section{Cultivos monozoospóricos y mantenimiento de los aislamientos}

La suspensión de zoosporas obtenida se usó para realizar diluciones sucesivas con el fin de obtener cultivos monozoospóricos y replicar los aislamientos en rodajas de papa en condiciones de humedad y temperatura mencionadas; 3 a 4 días, después fueron transferidos a medio agar-centeno (60 g de centeno, $15 \mathrm{~g}$ de agar y $20 \mathrm{~g}$ de sucrosa, más antibiótico) donde permanecieron durante dos meses aproximadamente. 


\section{Evaluación del tipo de apareamiento}

Cada uno de los aislamientos fue evaluado por dos metodologías en placas de Petri: sobre un medio agar-centeno (Método A) suplementado con $\beta$ sitosterol $0.05 \mathrm{~g} / \mathrm{l}$ (Figura 1), en rodajas de tubérculo de la variedad Tuquerreña (Método B). En ambas metodologías se utilizó micelio de un aislamiento universalmente avirulento (avrO), suministrado por el Centro Internacional de la Papa (CIP) como del tipo de apareamiento $A_{1}$ con cada uno de los aislamientos recolectados. Estas cajas se incubaron a $18^{\circ} \mathrm{C}$ en la oscuridad durante 8-14 días. Al cabo de este tiempo se evaluó la presencia o ausencia de oosporas en la interfase hifal, con la ayuda de microscopio de luz. Se realizaron 5 repeticiones por tratamiento utilizando como testigo, los apareamientos entre sí de cada aislamiento recolectado.

\section{Determinación in vitro de razas fisiológicas de $P$. infestans}

Para cada aislamiento se registró la reacción exhibida visualmente en los folíolos de 11 clones diferenciales de papa (con genes individuales para resistencia vertical a diferentes razas reconocidas del hongo), también suministrados por el CIP en presencia de cada aislamiento del patógeno a partir de inocules de $20 \mu \mathrm{l}$ de una solución que contenía entre 5,000 - 7,000 zoosporas $/ \mathrm{ml}$. El inóculo fue colocado en ambos lados de la nervadura central del envés del folíolo de cada clon diferencial y puesto dentro de a una placa de Petri invertido con agar nutritivo. Se realizaron ocho repeticiones por tratamiento, y se utilizó el cultivar Alfa como testigo.

De las plantas diferenciales de 30-50 días de sembradas, se inocularon e incubaron 4 folíolos bajo las condiciones de humedad y temperaturas mencionadas, durante 6 días en presencia de luz. Al cabo de este tiempo, se evaluó la respuesta de compatibilidad: (+) si se presentaba una lesión acompañada de esporulación del hongo; 0 una respuesta de hipersensibilidad $(H)$, cuando se observaron puntos necróticos dentro de la gota del inóculo (Figuras 1A y 1B).

La designación de las razas de $P$. infestans se realizó usando la nomenclatura propuesta por Black et al (1953) y un clon diferencial como testigo sin genes mayores sugerido por Tooley et al. (1986). 
Además se utilizó la nomenclatura octal para resumir la información obtenida de las interacciones entre cada uno de los aislamientos con las plantas diferenciales de papa (Goodwin et al., 1995).
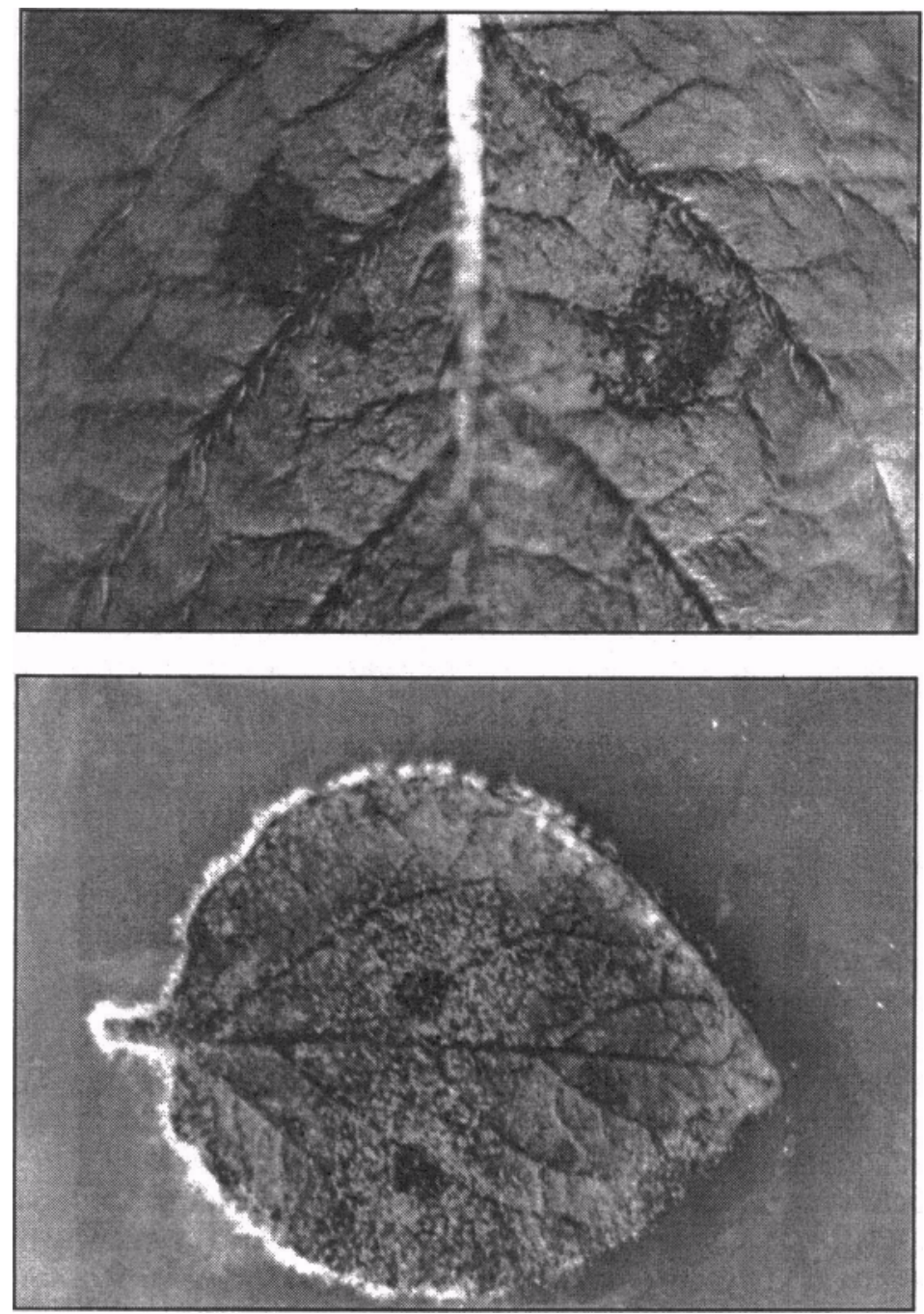

Figura 1. Reacción de compatibilidad (A) y de hipersensibilidad (B) entre un folíolo de un clon diferencial de papa con un aislamiento de $\mathrm{P}$. infestans. 


\section{Resultados}

Se observó diferencias en el crecimiento de las colonias del hongo tanto en medio agar-centeno (Método A) como en rodajas de papa (Método B). El método $B$ fue excelente para incrementar los aislamientos de $P$. infestans, ya que con él se obtuvo un rápido crecimiento micelial acompañado de una alta producción de esporangios.

Al evaluar y comparar todos los apareamientos de los diferentes aislamientos con el aislamiento testigo y de cada aislamiento consigo mismo, en los métodos propuestos, no se observó la formación de esporas sexuales (oosporas). En algunos apareamientos entre el aislamiento $U_{2}$ y el $A_{1}$ realizados sobre rodajas del tubérculo se observaron estructuras semejantes a oogonios.

Se observó una compatibilidad parcial $\left(C_{p}\right)$ entre algunos aislamientos y el diferencial con el genotipo $R_{2}$ (Tabla 2). Algunas veces se evidenció una hipersensibilidad de punto necrótico $(\mathrm{H}$.) en interacciones entre aislamientos y los diferenciales $R_{4}, R_{5}, R_{6}, R_{8}$ y $R_{9}$. Todos los aislamientos mostraron compatibilidad (+) con el cultivar $\alpha$, y los clones $R_{5}$ y $R_{9}$ siempre mostraron respuesta hipersensible con todos los aislamientos de $P$. infestans (Tabla 2).

Dentro de los 19 aislamientos de $P$. infestans evaluados por su virulencia con los 11 clones diferenciales de papa, se encontraron 6 patotipos diferentes, siendo la raza 7446 , la que se presentó con mayor frecuencia $(57.9 \%)$ seguida por la 5446 y la 7466 . Las otras razas, 7546, 1447 y 7046 fueron las de menor frecuencia (5.2\%) (Tabla 3).

El número promedio de factores de virulencia mostrado por la población del hongo fue 6.6 (Tabla 4). Cada uno de los aislamientos presentó al menos 5 factores de virulencia específica, con un máximo de 8 factores (Tabla 3). Los factores $r_{3}, r_{7}, r_{10}$ y $r_{11}$ siempre estuvieron presentes en todos los aislamientos.

La mayor complejidad (8 factores de virulencia) correspondió a los aislamientos $\mathrm{U}_{3}, \mathrm{UN}_{8}$ y $\mathrm{SV}_{7}$, mientras que el aislamiento procedente de Solanum muricatum presentó la menor complejidad (5 factores de virulencia) (Tabla 2).

Al realizar evaluaciones de patogenicidad con algunos aislamientos monozoospóricos, éstos presentaron los mismos factores de virulencia que se encontraban en sus respectivos aislamientos originales (Tabla 2). 
Tabla 2. Registro de la evaluación de los aislamientos de P. infestans con fenotipos diferenciales de papa.

\begin{tabular}{|c|c|c|c|c|c|c|c|c|c|c|c|c|}
\hline Aislamientos & & & & noti & os & ferer & ciale & de & Solá & um & & \\
\hline & $\alpha$ & $\mathrm{R}_{1}$ & $\mathrm{R}_{2}$ & $\mathrm{R}_{3}$ & $\mathrm{R}_{4}$ & $\mathrm{R}_{5}$ & $\mathrm{R}_{6}$ & $\mathrm{R}_{7}$ & $\mathrm{R}_{8}$ & $\mathrm{R}_{9}$ & $\mathrm{R}_{10}$ & $\mathrm{R}_{11}$ \\
\hline $\mathrm{U}_{1}$ & + & + & $\mathrm{C}_{\mathrm{p}}$ & + & + & $\mathrm{H}$ & $\mathrm{H}$. & + & $\mathrm{H}$ & $\mathrm{H}$ & + & + \\
\hline $\mathrm{U}_{2}$ & + & + & + & + & + & $\mathrm{H}$ & $\mathrm{H}$ & + & $\mathrm{H}$ & $\mathrm{H}$ & + & + \\
\hline $\mathrm{U}_{3}$ & + & + & $C_{p}$ & + & + & $\mathrm{H}$ & $\mathrm{H}$. & + & + & $\mathrm{H}$ & + & + \\
\hline $\mathrm{C}_{4}$ & + & + & $\mathrm{C}_{\mathrm{p}}$ & + & + & $\mathrm{H}$ & $\mathrm{H}$. & + & $\mathrm{H}$ & $\mathrm{H}$ & + & + \\
\hline $\mathrm{G}_{5}$ & + & + & $\mathrm{C}_{\mathrm{p}}$ & + & + & $\mathrm{H}$ & $\mathrm{H}$ & + & $\mathrm{H}$ & $\mathrm{H}$ & + & + \\
\hline $\mathrm{UN}_{6}$ & + & + & $\mathrm{H}$. & + & + & $\mathrm{H}$ & $\mathrm{H}$ & + & $\mathrm{H}$. & $\mathrm{H}$ & + & + \\
\hline $\mathrm{UN}_{8}$ & + & + & $\mathrm{C}_{\mathrm{p}}$ & + & + & $\mathrm{H}$ & $\mathrm{H}$ & + & + & $\mathrm{H}$ & + & + \\
\hline $\mathrm{SV}_{7}$ & + & + & + & + & + & $\mathrm{H}$ & + & + & $\mathrm{H}$. & $\mathrm{H}$ & + & + \\
\hline $\mathrm{E}_{9}$ & + & + & $\mathrm{C}_{\mathrm{p}}$ & + & + & $\mathrm{H}$ & $\mathrm{H}$ & + & $\mathrm{H}$ & $\mathrm{H}$ & + & + \\
\hline$E_{10}$ & + & + & + & + & + & $\mathrm{H}$ & $\mathrm{H}$. & + & $\mathrm{H}$ & $\mathrm{H}$ & + & + \\
\hline $\mathrm{SP}_{11}$ & + & + & $C_{p}$ & + & + & $\mathrm{H}$ & $\mathrm{H}$. & + & $\mathrm{H}$ & $\mathrm{H}$ & + & + \\
\hline $\mathrm{SP}_{12}$ & + & + & $\mathrm{C}_{\mathrm{p}}$ & + & + & $\mathrm{H}$ & $\mathrm{H}$ & + & $\mathrm{H}$ & $\mathrm{H}$ & + & + \\
\hline $\mathrm{ER}_{13}$ & + & + & $\mathrm{C}_{\mathrm{p}}$ & + & + & $\mathrm{H}$ & $\mathrm{H}$ & + & $\mathrm{H}$ & $\mathrm{H}$ & + & + \\
\hline $\mathrm{SR}_{14}$ & + & + & $\mathrm{C}_{\mathrm{p}}$ & + & + & $\mathrm{H}$ & $\mathrm{H}$ & + & $\mathrm{H}$ & $\mathrm{H}$ & + & + \\
\hline $\mathrm{SR}_{15}$ & + & + & $\mathrm{C}_{\mathrm{p}}$ & + & + & $\mathrm{H}$ & $\mathrm{H}$ & + & $\mathrm{H}$. & $\mathrm{H}$ & + & + \\
\hline $\mathrm{SS}_{16}$ & + & + & $\mathrm{H}$ & + & + & $\mathrm{H}$ & $\mathrm{H}$ & + & $\mathrm{H}$ & $\mathrm{H}$ & + & + \\
\hline $\mathrm{SS}_{17}$ & + & + & $\mathrm{H}$. & + & + & $\mathrm{H}$. & $\mathrm{H}$ & + & $\mathrm{H}$ & $\mathrm{H}$ & + & + \\
\hline $\mathrm{P}_{18}$ & + & + & $\mathrm{H}$. & + & + & $\mathrm{H}$. & $\mathrm{H}$. & + & $\mathrm{H}$. & $\mathrm{H}$. & + & + \\
\hline $\mathrm{T}_{19}$ & + & + & + & + & $\mathrm{H}$. & $\mathrm{H}$ & $\mathrm{H}$. & + & $\mathrm{H}$. & $\mathrm{H}$. & + & + \\
\hline $\mathrm{U}_{1} \mathrm{MZ}_{20}$ & + & + & $C_{p}$ & + & + & $\mathrm{H}$ & $\mathrm{H}$ & + & $\mathrm{H}$ & $\mathrm{H}$ & + & + \\
\hline $\mathrm{Sp}_{12} \mathrm{M} \mathrm{Z}_{21}$ & + & + & $\mathrm{C}_{\mathrm{p}}$ & + & + & $\mathrm{H}$ & $\mathrm{H}$ & + & $\mathrm{H}$ & $\mathrm{H}$ & + & + \\
\hline $\mathrm{SP}_{12} \mathrm{MZ}_{22}$ & + & + & $C_{p}$ & + & + & $\mathrm{H}$. & $\mathrm{H}$ & + & $\mathrm{H}$ & $\mathrm{H}$ & + & + \\
\hline
\end{tabular}

$(+)=$ Compatible; $\mathrm{Cp}=$ Compatibilidad parcial; $\mathrm{H}=$ hipersensibilidad $\mathrm{H} .=$ hipersensibilidad punto necrótico. 
Tabla 3. Frecuencia de razas fisiológicas de P. infestans obtenidas en diferenciales de Solanum tuberosum.

\begin{tabular}{lcccc}
\hline \multicolumn{1}{c}{$\begin{array}{c}\text { Aislamiento de } P . \\
\text { infestans* }\end{array}$} & Patotipo & N.octal & $\begin{array}{c}\text { Factores de } \\
\text { virulencia }\end{array}$ & \% Frecuencia \\
\hline $\mathrm{U}_{1} \mathrm{U}_{2} \mathrm{C}_{4} \mathrm{G}_{5} \mathrm{E}_{9} \mathrm{E}_{10}$ & $1.2 .3 . .4 .7 .10 .11$ & 7446 & 7 & 57.9 \\
$\mathrm{SP}_{11} \mathrm{SP}_{12} \mathrm{ER}_{13}$ & 1.3 .4 .7 .10 .11 & 5446 & 6 & 15.8 \\
$\mathrm{SR}_{14} \mathrm{SR}_{15}$ & 1.2 .3 .4 .7 .8 .10 .11 & 7466 & 8 & 10.5 \\
$\mathrm{UN}_{6} \mathrm{SS}_{16} \mathrm{SS}_{17}$ & 1.2 .3 .4 .6 .7 .10 .11 & 7546 & 8 & 5,2 \\
$\mathrm{UN}_{3} \mathrm{UN}_{8}$ & 3.4 .7 .10 .11 & 1446 & 6 & 5.2 \\
$\mathrm{SV}_{7}$ & 15.3 .7 .10 .11 & 7046 & 6 & 5.2 \\
$\mathrm{P}_{18}$ & & & & \\
$\mathrm{~T}_{19}$ & &
\end{tabular}

*Nomenclatura aislamientos ver Tabla 1.

Tabla 4. Frecuencias de factores de virulencia en colecciones de $\mathrm{P}$. infestans en diferentes regiones geográficas del mundo

\begin{tabular}{lcc}
\hline Región geográfica & $\mathrm{N}^{\circ}$ & Promedio factores virulencia \\
\hline Antioquia (Colombia) : & 19 & 6.6 \\
Valle Toluca (México) & 16 & 5.6 \\
Holanda & 14 & 4.1 \\
Reino Unido. & 61 & 3.7 \\
Estados Unidos, Canadá & 14 & 1.9 \\
Perú & 33 & 1.0 \\
\hline
\end{tabular}

Adaptado de Fry y Spielman (1991).

\section{Discusión}

\section{Evaluación del tipo de apareamiento}

La ausencia de oosporas en los apareamientos con aislamientos que fueron recolectados en las principales zonas paperas del Departamento de Antioquia, es un indicativo de que la población del hongo presente en esta zona corresponde a un solo tipo de apareamiento $\left(A_{1}\right)$. Esto presupone una reproducción asexual de $P$. infestans en esta región. 
Los apareamientos entre aislamientos de $P$. infestans generalmente se han realizado en medios de cultivos artificiales, pero muy pocas veces estos resultados han sido replicados en tejidos vivos. Las estructuras sexuales también han sido observadas en tubérculos de papa afectados por tizón, recolectados de cultivos atacados por el hongo (Smorawski, 1890; Lohnis, 1922; De Bruyn, 1923; Kotila, 1949). Lo anterior, sumado a los resultados obtenidos en el presente trabajo, hacen de las rodajas de papa (Método B) un sustrato fácil de conseguir y manejar para cultivar $P$. infestans, y para la realización de pruebas in vitro que permitan la detección de reproducción sexual en aislamientos de $P$. infestans.

\section{Composición de las razas fisiológicas de $P$. infestans}

La complejidad, en términos de número promedio de factores de virulencia específica encontrada en la población de $P$. infestans proveniente de las principales zonas paperas de Antioquia, es una de las más altas (6.6) que se ha reportado en el mundo (Tabla 4) (Tooley et al., 1986; Rivera - Peña, 1988). Contradictoriamente, la alta complejidad detectada en esta región está relacionada con una baja diversidad genética mostrada por los diferentes aislamientos.

Los estudios iniciales de reconocimiento de razas fisiológicas de $P$. infestans realizados en Colombia mostraron una alta correlación entre la complejidad de las razas y las variedades de papa comúnmente cultivadas, de modo que los factores de virulencia en el patógeno parecían estar influenciados por la existencia de genes mayores de resistencia al tizón en la papa (De Rojas, 1953; Guzmán, 1962). Sin embargo, estos estudios sólo contaron con un pequeño grupo de clones diferenciales de la papa, lo cual no permitía evaluar el promedio real de factores de virulencia presentes en las poblaciones del patógeno.

Se esperaría que la complejidad de los factores de virulencia del hongo estuviera determinada por los factores de resistencia presentes en las variedades de papa más comúnmente cultivadas en Colombia. Los resultados muestran que los factores de virulencia de $P$. infestans no correspondían en su totalidad con sus factores de resistencia equivalentes en las plantas hospedantes, a pesar de que algunos aislamientos del hongo habían sido recolectados de hospedantes sin genes mayores $(R)$ como $S$. phureja, S. muricatum y L. esculentum (Tabla 3).

Se podría considerar que la selección impuesta por los genotipos de las plantas huéspedes no sería un factor determinante para la 
complejidad en los factores de virulencia de las poblaciones de $P$. infestans en Antioquia. No obstante, sí parece ser un factor de selección importante en países como el Perú donde el cultivo intensivo de variedades de papa con el gen $R_{1}$ parece haber contribuido a la alta frecuencia de la raza aun de $P$. infestans (Tooley et al., 1989).

Además, las respuestas similares exhibidas en factores de virulencia por algunos aislamientos monozoospóricos con respecto a sus aislamientos madres, evaluados en la presente investigación, tienden a mostrar una alta uniformidad genética simultáneamente con una gran complejidad, similar a lo mostrado por estudios moleculares con isoenzimas (Tooley et al., 1985) y evaluaciones de mezclas de razas (Leachy Rich, 1969).

La correspondencia incompleta entre factores de resistencia en las plantas hospedantes y factores de virulencia en la población de $P$. infestans evaluada en el presente trabajo, puede estar influenciada por otras fuentes adicionales de variación tales como la herencia citoplasmática y la parasexualidad (Leach y Rich, 1969), fenómenos que permitirían la expresión de nuevos patotipos y de fenotipos recesivos que han estado ocultos por alelos dominantes.

A pesar de que en la población de $P$. infestans evaluada en este trabajo se encontraron 6 razas fisiológicas distintas, sus diferencias radican solamente en 1-3 factores de virulencia, con ausencia de los factores aur ${ }_{5}$ y $r_{9}$. Esto permite suponer que una raza fisiológica de alta complejidad, tiende a predominar en un huésped específico.

La complejidad parcial detectada en el diferencial $R_{2}$ se puede deber a la presencia de genes responsables de resistencia horizontal (cuantitativa), presentes en este diferencial (Turkensteen, 1989) y no a un efecto de expresividad variable exhibido por el $\mathrm{r}_{2}$.

La alta complejidad detectada en los aislamientos de $P$. infestans recolectados en las diferentes regiones de Antioquia, sugieren la utilización de resistencia horizontal para futuros programas de mejoramiento genético de la papa, tendientes a obtener variedades resistentes a este patógeno y a otros agentes con comportamiento genético similar. 


\section{Referencias Bibliográficas}

1. Black, W.; Mastenbroek, C.; Mills, W.R.; Paterson, L.C. 1953. A proposal for an international nomenclatura of races of Phytophthora infestans and genes controlling immunity in Solanum demisum derívales. Euphytica 2:173-179.

2. Buriticá, C.P. 1994. Ciclo de vida de los patógenos de las plantas cultivadas en el trópico. Ascolfi informa 20 (1) :5-12.

3. Daggett, S.S; Götz, E.; Therrien, C.D. 1983. Phenotypic changes in populations of Phytophthora infestans from Eastern Germany. Phytopathology 83:319-323.

4. De Rojas, P.E. 1953. El problema de las razas fisiológicas de Phytophthora infestans (Moni.) de Bary. Contribución a su estudio. Información Técnica 1(1): 1-49.

5. De Bruyn, H.L.G. 1923. The oospores of Phytophthora infestans. Rept. Intern. Conf. Phytopathol. and Econ. Entomol. Holland p. 30-31.

6. Fry, W.E.; Spielman, DJ. 1991. Population Biology. En: Advances in Plant Pathology. Vol. 7 Ingram, D.S. and Williams, P.H. (eds.) Phytophthora infestans, the cause of late blight of potato. London. 273 p. 171-192p.

7. Fry, W.E.; Tooley, P.W.; Spielman, L.J. 1989. The importance of the perfect stage of Phytophthora infestans from the standpoint of epidemiology and adaptation. p. 17-30. En : Report of the planning conference of fungal diseases of potato. Lima. International Potato Center. 17-30p.

8. Galindo, J.; Gallegly, M.E. 1960. The nature of sexuality in Phytophthora infestans. Phytopathology $50: 123-128$.

9. Gallegly, M.E.; Galindo, J. 1958. Mating types and oospores of Phytophthora infestans in nature in Mexico. Phytopathology $48: 274-277$

10. Goodwin, S.B.; Sujkowsky, L.S.; Fry, W.G. 1995. Rapid evolution of pathogenicity within clonal lineages of the potato late blight disease fungus. Phytopathology 85 : 669-675.

11. Guzman, N.J. 1962. La gota de la papa Phytophthora infestans (Mont.) de Bary. ICA Informe Anual de Labores. Programa Nacional de Fitopatología. 20-24 p..

12. Henfling, J.W. 1987. Late Blight of potato (Phytophthora infestans). International Potato Center. Lima, Perú. p. 4-24. 
13. Hohl, H.R.; Iselin, K. 1984. Strains of Phytophthora infestans from Switzerland with $\mathrm{A}_{2}$ mating type behavior. Trans. Br. Mycol. Soc. 83: 529-530.

14. Ko, W. H. 1994. An alternative possible origin of the A2 mating type of Phytopthora infestans outside of México. Phytopathology 84:12241227.

15. Kotila, M.A. 1949. Studies on the potato blight fungus, Phytophthora infestans (Mont.) de Bary. MS Tesis, Univ. of Minnesota. $35 \mathrm{p}$.

16. Leach, S.S.; Ritch, A.E. 1969 The possible role of parasexuality and cytoplasmic variation in race diferentiation in Phytophthora infestans. Phytopathology $59: 1360-1365$.

17. Lohnis, M.D. 1922. Onderzock over Phytophthora infestans (Mont.) de Bary op de aardappelplant. H. Veenman, Wageningen, Holland. 96 p.

18. Malcolmson, J.F. 1969. Races of Phytophthora infestans ocurring in Great Britain. Transactions of the British Mycological Society 53 :417423.

19. Niederhauser, J.S.; Mills, W.R. 1953. Resistance of Solanum especies to Phytophthora infestans in México. Phytopathology 43:456-457.

20. Rivera-Peña, A. 1988. Interaction between wild tuber-bearing solanum species and Phytophthora infestans (Mont.) de Bary in a natural habitat and implication of the results for potato breeding. PhD disertation. Swedish University of Agricultural Sciences, Upsala, Sweden.

21. Shattock, R.C.; Shaw, D.S.; Fyfe, A.M.; Dunn, J.R.; Loney, K.; Shattock, J.A. 1990. Phenotypes of Phytophthora infestans collected in England and Wales from 1985-1988. Mating type, response to metalaxyl and isozyme analysis. Plant Pathology 39 :242-248.

22. Shaw, D.S. 1987. The breding system of Phytophthora infestans: The role of A2 mating type. Genetics and Plant Pathogenesis. P.R. Day, and G. Jells (eds). Black WII Scientific Publications, Oxford. 151-174 p.

23. Smorawsky, J. 1890. Zur entwicklugsgeschichte der Phytophthora infestans. Landwirstsch Jahrb 19:1-12 
24. Tooley, P.W; Fry, W.E; Villareal, MJ. 1985. Isozyme characterization of sexual and asexual Phytophthora infestans populations. Journal of Heredity $76: 431-435$.

25. Tooley, P.W.; Sweigard, J.A.; Fry, W.E. 1986. Fitness and virulence of Phytophthora infestans isolates from sexual and asexual populations. Phytopathology $76: 1209-1212$.

26. Tooley, P.W.; Therrien. C.D.; Ritch, D.L. 1989. Mating type, race composition, nuclear DNA cont and isozyme analysis of peruvian isolates of Phytophthora infestans. Phytopathology 79:478-481.

27. Turkensteen, L. J. 1989. Interaction of R-genes in breeding for resistance of potatoes against Phytophthora infestans. En: Report of the planning conference on fungal diseases of the potato. Lima, International of Potato Center. p. 85-96.

28. Zachman, R. 1976. Investigation on the ocurrence of the perfect stage of Phytophthora infestans in Perú. Phytopathology $11:$ 85-86. 